\title{
Intuitionistic fuzzy actions
}

\section{S. Melliani, M. Elomari, I. Bakhadach and L. S. Chadli}

\author{
Department of Mathematics \\ Sultan Moulay Slimane University \\ LMACS, Laboratoire de Mathématiques Appliquées \& Calcul Scientifique \\ PO Box 523, 23000 Beni Mellal, Morocco \\ e-mails: said.melliani@gmail.com \\ m.elomari@gmail.com \\ idris.bakhadach@gmail.com \\ sa.chadli@yahoo.fr
}

Received: 7 April 2018

Revised: 20 October 2018

Accepted: 25 October 2018

\begin{abstract}
In this paper is we will try to generalizes actions of a group on a set to deal with situations where imprecision and uncertainty are present. Intuitionistic Fuzzy actions can handle the granularity of a set or even create it by defining an intuitionistic fuzzy equivalence relation on it.
\end{abstract}

Keywords: Intuitionistic fuzzy subgroups, Intuitionistic fuzzy relations, $(T, S)$-indistinguishability.

2010 Mathematics Subject Classification: 03E72.

\section{Introduction}

In 1983, Atanassov introduced the theory of intuitionistic fuzzy sets (see [1, 2]), which plays important role in modern mathematics in general as it represents a generalization of fuzzy sets.

The authors in [4] introduced the notion of the intuitionistic fuzzy groups based on the intuitionistic fuzzy space. In [3] the notion of fuzzy actions is presented with its properties. From this idea and taking into account the generalization of the intuitionistic fuzzy theory we will give sense of the notion of action of a group on a set in the intuitionistic fuzzy frame, which is a very useful tool in many branches of mathematics and computer science. 


\section{Preliminaries}

Definition 1. (Triangular norm) A t-norm is any monotonous, commutative, associative mapping $T:[0,1] \times[0,1] \longrightarrow[0,1]$ satisfying $T(1, x)=x$ for all $x \in[0,1]$.

Definition 2. (Triangular Conorm) A t-conorm is any monotonous, commutative, associative mapping $S:[0,1] \times[0,1] \longrightarrow[0,1]$ satisfying $S(0, x)=x$ for all $x \in[0,1]$.

Note that a fuzzy relation on any set $X$ is a fuzzy set $\mu: X \times X \longrightarrow[0,1]$.

Definition 3. [7] An intuitionistic fuzzy set $A=\left(\mu_{A}, \nu_{A}\right)$ is called an intuitionistic fuzzy relation on $X$ if $\mu_{A}$ and $\nu_{A}$ are fuzzy relations on $X$ with $0 \leq \mu_{A}+\nu_{A} \leq 1$.

Definition 4. [5] An intuitionistic fuzzy relation $E=\left(E_{1}, E_{2}\right)$ on a set $X$ (i.e., $\left.E: X \times X \rightarrow L^{*}\right)$, $L^{*}=\{(x, y), 0 \leq x+y \leq 1\}$, is a $(T, S)$-indistinguishability operator on $X$ if and only for all $x, y, z$ of $X$ satisfies the following properties.

1. $E_{1}(x, x)=1$ and $E_{2}(x, x)=0$ (Reflexivity)

2. $E_{1}(x, y)=E_{1}(y, x)$ and $E_{2}(x, y)=E_{2}(y, x)$ (Symmetry)

3. $T\left(E_{1}(x, y), E_{1}(y, z)\right) \leq E_{1}(x, z)$ and $S\left(E_{2}(x, y), E_{2}(y, z)\right) \geq E_{2}(x, z)$ (Transitivity).

$(T, S)$-indistinguishability operators extend the concept of equivalence relation and equality to the intuitionistic fuzzy framework and they are also called intuitionistic fuzzy $(T, S)$ equivalences and intuitionistic fuzzy $(T, S)$-equality relations. We define the sup- $(T, S)$-indistinguishability operators product between intuitionistic fuzzy relations that will be needed in the study of intuitionistic fuzzy mappings.

Definition 5. Let $X, Y, Z$ be sets and $R: X \times Y \rightarrow L^{*}$ and $R^{\prime}: Y \times Z \rightarrow L^{*}$ intuitionistic fuzzy relations. The sup-(T,S) product $R_{\mathrm{O}_{T, S}} R^{\prime}$ of $R$ and $R^{\prime}$ is the intuitionistic fuzzy relation $R_{\mathrm{o}_{T, S}} R^{\prime}: X \times Z \rightarrow L^{*}$ defined for all $x \in X, z \in Z$ by

$$
\begin{aligned}
& \mu_{R_{T, S} R^{\prime}}(x, z)=\sup _{y \in Y} T\left(R_{1}(x, y), R_{1}^{\prime}(y, z)\right), \\
& \nu_{R \mathrm{o}_{T, S} R^{\prime}}(x, z)=\inf _{y \in Y} S\left(R_{2}(x, y), R_{2}^{\prime}(y, z)\right) .
\end{aligned}
$$

Intuitionistic fuzzy mappings generalize the concept of mapping between two sets $X$ and $Y$. The sets are supposed to be endowed with $(T, S)$-indistinguishability operators, and compatibility of the fuzzy mappings with them is imposed.

Definition 6. Let $E_{X}$ and $E_{Y}$ be $(T, S)$-indistinguishability operators on two sets $X$ and $Y$, respectively. $R: X \times Y \rightarrow L^{*}$ is an intuitionistic fuzzy mapping from $X$ onto $Y$ if and only iffor all $x, x^{\prime} \in X$ and for all $y, y^{\prime} \in Y$

$$
\begin{aligned}
& T\left(R_{1}(x, y), E_{X_{1}}\left(x, x^{\prime}\right), E_{Y_{1}}\left(y, y^{\prime}\right)\right) \leq R_{1}\left(x^{\prime}, y^{\prime}\right) \\
& S\left(R_{2}(x, y), E_{X_{2}}\left(x, x^{\prime}\right), E_{Y_{2}}\left(y, y^{\prime}\right)\right) \geq R_{2}\left(x^{\prime}, y^{\prime}\right)
\end{aligned}
$$




$$
\begin{aligned}
& T\left(R_{1}(x, y), R_{1}(x, y)\right) \leq E_{Y_{1}}\left(y, y^{\prime}\right) \\
& S\left(R_{2}(x, y), R_{2}(x, y)\right) \geq E_{Y_{2}}\left(y, y^{\prime}\right)
\end{aligned}
$$

$R$ is perfect if and only if for all $x \in X$ there exists $y \in Y$ such that $R(x, y)=1_{L^{*}}$. i.e., $R_{1}(x, y)=1$ and $R_{2}(x, y)=0$.

Definition 7. Let $E_{X}$ and $E_{Y}$ be $(T, S)$-indistinguishability operators on two sets $X$ and $Y$, respectively. An intuitionistic fuzzy mapping $R$ from $X$ onto $Y$ is injective if and only if for all $x$, $x^{\prime} \in X$ and for all $y, y^{\prime} \in Y$

$$
\begin{aligned}
& T\left(R_{1}(x, y), R_{1}\left(x^{\prime}, y^{\prime}\right), E_{Y_{1}}\left(y, y^{\prime}\right)\right) \leq E_{X_{1}}\left(x, x^{\prime}\right) \\
& S\left(R_{2}(x, y), R_{2}\left(x^{\prime}, y^{\prime}\right), E_{Y_{2}}\left(y, y^{\prime}\right)\right) \geq E_{X_{2}}\left(x, x^{\prime}\right)
\end{aligned}
$$

Definition 8. Let $E_{X}=\left(E_{X_{1}}, E_{X_{1}}\right)$ and $E_{Y}=\left(E_{Y_{1}}, E_{Y_{2}}\right)$ be $(T, S)$-indistinguishability operators on two sets $X$ and $Y$, respectively. Given an intuitionistic fuzzy mapping $R$ from $X$ onto $Y$, the image of $R(\operatorname{Im}(R))$ is given by

$$
\begin{aligned}
& \mu_{I m}(y)=\sup _{x \in X, y^{\prime} \in Y} T\left(R_{1}\left(x, y^{\prime}\right), E_{Y_{1}}\left(y^{\prime}, y\right)\right) \\
&=\sup _{x \in X}\left(\nu_{R_{T}, S} R^{\prime}\right. \\
&
\end{aligned}
$$

and

$$
\begin{aligned}
\nu_{\mathrm{Im}}(y) & =\inf _{x \in X, y^{\prime} \in Y} S\left(R_{2}\left(x, y^{\prime}\right), E_{Y_{2}}\left(y^{\prime}, y\right)\right) \\
& =\inf _{x \in X}\left(\nu_{R_{T, S} R^{\prime}}(x, z)\right) .
\end{aligned}
$$

The infimum of the last expression for $y \in Y, \inf _{y \in Y}\{\operatorname{Im}(y)\}$, is the degree of surjectivity of $R$ is strongly surjective if and only if its degree of surjectivity is 1.

Definition 9. Let $R: X \times Y \rightarrow L^{*}$ and $R^{\prime}: Y \times Z \rightarrow L^{*}$ be two intuitionistic fuzzy mappings. The composition of $R$ and $R^{\prime}$ is the intuitionistic fuzzy mapping $M=R \circ R^{\prime}: X \times Z \rightarrow L^{*}$ defined for all $x \in X, z \in Z$ by

$$
M(x, z)=\left(R_{\mathrm{o}_{T, S}} E_{Y} \mathrm{O}_{T, S} R^{\prime}\right)(x, z)
$$

with

$$
\begin{aligned}
& M_{1}(x, z)=\sup _{y, y^{\prime} \in Y} T\left(R_{1}(x, y), E_{Y_{1}}\left(y, y^{\prime}\right), R_{1}^{\prime}\left(y^{\prime}, z\right)\right) \\
& M_{2}(x, z)=\inf _{y, y^{\prime} \in Y} S\left(R_{2}(x, y), E_{Y_{2}}\left(y, y^{\prime}\right), R_{2}^{\prime}\left(y^{\prime}, z\right)\right)
\end{aligned}
$$

Proposition 1. Let $R: X \times Y \rightarrow L^{*}$ and $R^{\prime}: Y \times Z \rightarrow L^{*}$ be two intuitionistic fuzzy mappings. If the composition mapping $M=R \mathrm{o} R^{\prime}$ is injective and $R^{\prime}$ is a perfect mapping, then $R$ is injective. 
Proof. Injectivity of $M$ means

$$
\begin{aligned}
& E_{X_{1}}\left(x, x^{\prime}\right) \geq T\left(M_{1}(x, z), M_{1}\left(x^{\prime}, z^{\prime}\right), E_{Z_{1}}\left(z, z^{\prime}\right)\right) \\
& E_{X_{2}}\left(x, x^{\prime}\right) \leq S\left(M_{2}(x, z), M_{2}\left(x^{\prime}, z^{\prime}\right), E_{Z_{2}}\left(z, z^{\prime}\right)\right)
\end{aligned}
$$

for all $x, x^{\prime}, z, z^{\prime} \in Z$. In particular, for $z^{\prime}=z$ we get

$$
\begin{aligned}
E_{X_{1}}\left(x, x^{\prime}\right) & \geq \sup _{y, y^{\prime}, y^{\prime \prime}, y^{\prime \prime \prime} \in Y} T(a, b, c, d, e, f, g) \\
& \geq \sup _{y, y^{\prime} \in Y} T\left(a, b, c, R_{1}\left(x^{\prime}, y^{\prime}\right), E_{Y_{1}}\left(y^{\prime}, y^{\prime}\right), R_{1}^{\prime}\left(y^{\prime}, z\right)\right),
\end{aligned}
$$

where $a=R_{1}(x, y), b=E_{Y_{1}}\left(y, y^{\prime}\right), c=R_{1}^{\prime}\left(y^{\prime}, z\right), d=R_{1}\left(x^{\prime}, y^{\prime \prime}\right), e=E_{Y_{1}}\left(y^{\prime \prime}, y^{\prime \prime \prime}\right)$, $f=R_{1}^{\prime}\left(y^{\prime \prime \prime}, z\right), g=E_{Z_{1}}(z, z)$ and

$$
\begin{aligned}
E_{X_{2}}\left(x, x^{\prime}\right) & \leq \inf _{y, y^{\prime}, y^{\prime \prime}, y^{\prime \prime \prime} \in Y} S\left(a^{\prime}, b^{\prime}, c^{\prime}, d^{\prime}, e^{\prime}, f^{\prime}, g^{\prime}\right) \\
& \leq \inf _{y, y^{\prime} \in Y} S\left(a^{\prime}, b^{\prime}, c^{\prime}, R_{2}\left(x^{\prime}, y^{\prime}\right), E_{Y_{2}}\left(y^{\prime}, y^{\prime}\right), R_{2}^{\prime}\left(y^{\prime}, z\right)\right)
\end{aligned}
$$

where $a^{\prime}=R_{2}(x, y), b^{\prime}=E_{Y_{2}}\left(y, y^{\prime}\right), c^{\prime}=R_{2}^{\prime}\left(y^{\prime}, z\right), d^{\prime}=R_{2}\left(x^{\prime}, y^{\prime \prime}\right), e^{\prime}=E_{Y_{2}}\left(y^{\prime \prime}, y^{\prime \prime \prime}\right)$, $f^{\prime}=R_{2}^{\prime}\left(y^{\prime \prime \prime}, z\right), g^{\prime}=E_{Z_{2}}(z, z)$.

The last inequality follows by considering $y^{\prime}=y^{\prime \prime}=y^{\prime \prime \prime}$. Now, since $R^{\prime}$ is perfect, for $y^{\prime}$ there exists $z_{y^{\prime}}$ with $R^{\prime}\left(y^{\prime}, z_{y^{\prime}}\right)=1_{L^{*}}$ and the last expression is greater (smaller) than or equal (respectively) to

$$
\begin{gathered}
T\left(R_{1}(x, y), R_{1}\left(x^{\prime}, y^{\prime}\right), E_{Y_{1}}\left(y, y^{\prime}\right)\right), \\
\left(R_{2}(x, y), R_{2}\left(x^{\prime}, y^{\prime}\right), E_{Y_{2}}\left(y, y^{\prime}\right)\right),
\end{gathered}
$$

which means injectivity of $R$.

Definition 10. Let $R: X \times Y \rightarrow L^{*}$ and $R^{\prime}: Y \times X \rightarrow L^{*}$ be two intuitionistic fuzzy mappings. $R$ is the inverse fuzzy mapping of $R_{1}$ (and vice versa) if and only if

$$
\begin{aligned}
& T\left(R_{1}^{\prime}(x, y), E_{Y_{1}}\left(y, y^{\prime}\right), R_{1}^{\prime}\left(y^{\prime}, x^{\prime}\right)\right) \leq E_{X_{1}}\left(x, x^{\prime}\right), \\
& S\left(R_{2}(x, y), E_{Y_{2}}\left(y, y^{\prime}\right), R_{2}^{\prime}\left(y^{\prime}, x^{\prime}\right)\right) \geq E_{X_{2}}\left(x, x^{\prime}\right), \\
& T\left(R_{1}^{\prime}(y, x), E_{X_{1}}\left(x, x^{\prime}\right), R_{1}\left(x^{\prime}, y^{\prime}\right)\right) \leq E_{Y_{1}}\left(y, y^{\prime}\right), \\
& S\left(R_{2}^{\prime}(y, x), E_{X_{2}}\left(x, x^{\prime}\right), R_{2}\left(x^{\prime}, y^{\prime}\right)\right) \geq E_{Y_{2}}\left(y, y^{\prime}\right) .
\end{aligned}
$$

Definition 11. Let $R$ be an intuitionistic fuzzy relation $R: X \times Y \rightarrow L^{*}$. The inverse relation $R^{\prime}$ of $R$ (usually denoted by $R^{-1}$ ) is the intuitionistic fuzzy relation $R^{\prime}: Y \times X \rightarrow L^{*}$ defined for all $x \in X, y \in Y$ by $R^{\prime}(y, x)=R(x, y)$.

Proposition 2. If $R: X \times Y \rightarrow L^{*}$ and $R^{-1}: Y \times X \rightarrow L^{*}$ are both intuitionistic fuzzy mappings, then

a) $R$ is the inverse mapping of $R^{-1}$. 
b) $R$ and $R^{-1}$ are injective.

Proof. a)

$$
\begin{aligned}
T\left(R_{1}(x, y), E_{Y_{1}}\left(y, y^{\prime}\right), R_{1}^{-1}\left(y^{\prime}, x^{\prime}\right)\right) & =T\left(R_{1}(x, y), E_{Y_{1}}\left(y, y^{\prime}\right), R_{1}\left(x^{\prime}, y^{\prime}\right)\right) \\
& \leq E_{X_{1}}\left(x, x^{\prime}\right) \\
T\left(R_{1}^{-1}(y, x), E_{X_{1}}\left(x, x^{\prime}\right), R_{1}\left(x^{\prime}, y^{\prime}\right)\right) & =T\left(R_{1}^{-1}(y, x), E_{X_{1}}\left(x, x^{\prime}\right), R_{1}\left(x^{\prime}, y^{\prime}\right)\right) \\
& \leq E_{Y_{1}}\left(y, y^{\prime}\right)
\end{aligned}
$$

and

$$
\begin{aligned}
S\left(R_{2}(x, y), E_{Y_{2}}\left(y, y^{\prime}\right), R_{2}^{-1}\left(y^{\prime}, x^{\prime}\right)\right) & =S\left(R_{2}(x, y), E_{Y_{2}}\left(y, y^{\prime}\right), R_{2}\left(x^{\prime}, y^{\prime}\right)\right) \\
& \geq E_{X_{2}}\left(x, x^{\prime}\right) \\
S\left(R_{2}^{-1}(y, x), E_{X_{2}}\left(x, x^{\prime}\right), R_{2}\left(x^{\prime}, y^{\prime}\right)\right) & =S\left(R_{2}^{-1}(y, x), E_{X_{2}}\left(x, x^{\prime}\right), R_{2}\left(x^{\prime}, y^{\prime}\right)\right) \\
& \geq E_{Y_{2}}\left(y, y^{\prime}\right) .
\end{aligned}
$$

b)

$$
\begin{aligned}
T\left(R_{1}(x, y), R_{1}\left(x^{\prime}, y^{\prime}\right), E_{Y_{1}}\left(y, y^{\prime}\right)\right) & =T\left(R_{1}(x, y), R_{1}^{-1}\left(y^{\prime}, x^{\prime}\right), E_{Y_{1}}\left(y, y^{\prime}\right)\right) \\
& \leq E_{X_{1}}\left(x, x^{\prime}\right) \\
T\left(R_{1}^{-1}(y, x), R_{1}^{-1}\left(y^{\prime}, x^{\prime}\right), E_{X_{1}}\left(x, x^{\prime}\right)\right) & =T\left(R_{1}^{-1}(y, x), R_{1}\left(x^{\prime}, y^{\prime}\right), E_{X_{1}}\left(x, x^{\prime}\right)\right) \\
& \leq E_{Y_{1}}\left(y, y^{\prime}\right)
\end{aligned}
$$

and

$$
\begin{aligned}
S\left(R_{2}(x, y), R_{2}\left(x^{\prime}, y^{\prime}\right), E_{Y_{2}}\left(y, y^{\prime}\right)\right) & =S\left(R_{2}(x, y), R_{2}^{-1}\left(y^{\prime}, x^{\prime}\right), E_{Y_{2}}\left(y, y^{\prime}\right)\right) \\
& \geq E_{X_{2}}\left(x, x^{\prime}\right) \\
S\left(R_{2}^{-1}(y, x), R_{2}^{-1}\left(y^{\prime}, x^{\prime}\right), E_{X_{2}}\left(x, x^{\prime}\right)\right) & =S\left(R_{2}^{-1}(y, x), R_{2}\left(x^{\prime}, y^{\prime}\right), E_{X_{2}}\left(x, x^{\prime}\right)\right) \\
& \geq E_{Y_{2}}\left(y, y^{\prime}\right) .
\end{aligned}
$$

This completes the proof.

\section{Intuitionistic fuzzy actions}

The concept of actions of a group fuzzified by Boixader and Recasens [3]. In [6], Tarsuslu et al. introduced intuitionistic fuzzy actions of a group on a set by extending definition to the lattice $[0,1] \times[0,1]$. In our study, we introduced this notion of intuitionistic fuzzy actions of a group on a set by using t-norm and t-conorm.

Definition 12. Let $G$ be a monoid with neutral element e and I be a non-empty set. $\alpha: G \times I \rightarrow I$ is an action of $G$ on $I$ if and only if for all $g, h \in G, x \in I$

1. $(h g) x=h(g x)$,

2. $e x=x$,

where $\alpha(g, x)$ is denoted by $g x$. 
Definition 13. Let $G$ be a monoid with neutral element e, $I$ be a non-empty set and $(T, S)$ be a t-norm. $\alpha: G \times I \times I \rightarrow L^{*}$ is a $(T, S)$-intuitionistic fuzzy action (or simply an intuitionistic fuzzy action) of $G$ on $I$ if and only if for all $g, h \in G, x \in I$

1. $T\left(\alpha_{1}(h g, x, y), \alpha_{1}(g, x, z)\right) \leq \alpha_{1}(h, z, y)$

2. $S\left(\alpha_{2}(h g, x, y), \alpha_{2}(g, x, z)\right) \geq \alpha_{2}(h, z, y)$

3. $T\left(\alpha_{1}(g, x, z), \alpha_{1}(h, z, y)\right) \leq \alpha_{1}(h g, x, y)$

4. $S\left(\alpha_{2}(g, x, z), \alpha_{2}(h, z, y)\right) \geq \alpha_{2}(h g, x, y)$

and $\alpha(e, x, x)=1_{L^{*}}$, i.e., $\alpha_{1}(e, x, x)=1$ and $\alpha_{2}(e, x, x)=0$.

Definition 14. An intuitionistic fuzzy action $\alpha$ is quasi-perfect if and only if for all $g \in G, x \in I$ there exists $y \in I$ such that $\alpha(g, x, y)=1_{L^{*}} . \alpha$ is perfect if and only if the previous $y$ is unique for each $g \in G$ and $x \in X$.

Lemma 1. Let $\alpha$ be an intuitionistic fuzzy action of $G$ on $X$. If for $g \in G$ and $x, y$, $y^{\prime} \in I \alpha(g, x, y)=\alpha\left(g, x, y^{\prime}\right)=1_{L^{*}}$, then $\alpha\left(e, y, y^{\prime}\right)=1_{L^{*}}$.

Proof. From 1., 2. of Definition 13

$$
\begin{aligned}
& 1=T\left(\alpha_{1}(g, x, y), \alpha_{1}\left(g, x, y^{\prime}\right)\right) \leq \alpha_{1}\left(e, y, y^{\prime}\right), \\
& 0=S\left(\alpha_{2}(g, x, y), \alpha_{2}\left(g, x, y^{\prime}\right)\right) \geq \alpha_{2}\left(e, y, y^{\prime}\right) .
\end{aligned}
$$

Given a quasi-perfect intuitionistic fuzzy action $\alpha$ of $G$ on $I$ we can consider the (crisp) equivalence relation $\sim$ on $I$ defined by $x \sim y$ if and only if $\alpha(e, x, y)=1_{L^{*}}$, and the fuzzy action $\alpha$ of $G$ on $\bar{I}=I / \sim$ defined by $\alpha(g, \mathrm{X}, y)=\alpha(g, x, y)$.

Proposition 3. Let $\alpha$ be an intuitionistic fuzzy action of $G$ on $X . \sim$ is an equivalence relation, $\bar{\alpha}$ is well defined and is an intuitionistic fuzzy action of $G$ on $\bar{I}=I / \sim$.

Proof.

Reflexivity: $x \sim x$, because $\alpha(e, x, x)=1_{L^{*}}$.

Symmetry:

$$
\begin{aligned}
& 1=\alpha_{1}(e, x, y)=T\left(\alpha_{1}(e, x, x), \alpha_{1}(e, x, y)\right) \leq \alpha_{1}(e, y, x) \\
& 0=\alpha_{2}(e, x, y)=S\left(\alpha_{2}(e, x, x), \alpha_{2}(e, x, y)\right) \geq \alpha_{2}(e, y, x)
\end{aligned}
$$

by Property 1) and 2).

Transitivity: $x \sim y$ and $y \sim z$, then

$$
\begin{aligned}
& 1=T\left(\alpha_{1}(e, x, y), \alpha_{1}(e, y, z)\right) \leq \alpha_{1}(e, x, z) \\
& 0=S\left(\alpha_{2}(e, x, y), \alpha_{2}(e, y, z)\right) \geq \alpha_{2}(e, x, z)
\end{aligned}
$$


by Property 3) and 4).

In order to prove that $\bar{\alpha}$ is well defined, we must show that if

$$
\begin{aligned}
& \alpha\left(e, x, x^{\prime}\right)=1_{L^{*}} \text { and } \alpha\left(e, y, y^{\prime}\right)=1_{L^{*}}, \text { then } \alpha(g, x, y)=\alpha\left(g, x^{\prime}, y\right) \\
& \begin{aligned}
\alpha_{1}(g, x, y) & =T\left(\alpha_{1}(g, x, y), \alpha_{1}\left(e, x, x^{\prime}\right) \alpha_{1}\left(e, y, y^{\prime}\right)\right) \\
& =T\left(\alpha_{1}(g, x, y), \alpha_{1}\left(e, x^{\prime}, x\right), \alpha_{1}\left(e, y, y^{\prime}\right)\right) \\
& \leq \alpha_{1}\left(g, x^{\prime}, y^{\prime}\right)
\end{aligned}
\end{aligned}
$$

by symmetry we have $\alpha_{1}(g, x, y)=\alpha_{1}\left(g, x^{\prime}, y\right)$. And we have

$$
\begin{aligned}
\alpha_{2}(g, x, y) & =S\left(\alpha_{2}(g, x, y), \alpha_{2}\left(e, x, x^{\prime}\right) \alpha_{2}\left(e, y, y^{\prime}\right)\right) \\
& =T\left(\alpha_{2}(g, x, y), \alpha_{2}\left(e, x^{\prime}, x\right), \alpha_{2}\left(e, y, y^{\prime}\right)\right) \\
& \geq \alpha_{2}\left(g, x^{\prime}, y^{\prime}\right)
\end{aligned}
$$

By symmetry we have $\alpha_{2}(g, x, y)=\alpha_{2}\left(g, x^{\prime}, y\right)$. Then $\alpha(g, x, y)=\alpha\left(g, x^{\prime}, y\right)$. It is straightforward to prove that $\bar{\alpha}$ is an intuitionistic fuzzy action.

This result allows us to restrict the study of quasi-perfect intuitionistic fuzzy actions to perfect ones.

Proposition 4. If $\alpha$ is a perfect intuitionistic fuzzy action of $G$ on $I$ and, for $g \in G$ and $x \in I, x_{g} \in I$ is the unique element of I such that $\alpha\left(g, x, x_{g}\right)=1_{L^{*}}$, then $g x=x_{g}$ is a crisp action.

Proof. Straightforward.

Reciprocally, it will be shown in Proposition 13 how to fuzzify crisp actions to obtain perfect intuitionistic fuzzy ones. From now on, we will assume that $G$ is a group.

Lemma 2. If $\alpha$ is an intuitionistic fuzzy action of $G$ on $I, h \in G$ and $x, z \in I$, then $\alpha\left(h^{-1}, x, z\right)=\alpha(h, z, x)$.

Proof. From 1. and 2. in Definition 13

$$
T\left(\alpha_{1}\left(h h^{-1}, x, y\right), \alpha_{1}\left(h^{-1}, x, z\right)\right) \leq \alpha_{1}(h, z, y)
$$

and

$$
S\left(\alpha_{2}\left(h h^{-1}, x, y\right), \alpha_{2}\left(h^{-1}, x, z\right)\right) \geq \alpha_{2}(h, z, y)
$$

or

$$
T\left(\alpha_{1}(e, x, y), \alpha_{1}\left(h^{-1}, x, z\right)\right) \leq \alpha_{1}(h, z, y)
$$

and

$$
S\left(\alpha_{2}(e, x, y), \alpha_{2}\left(h^{-1}, x, z\right)\right) \geq \alpha_{2}(h, z, y) .
$$


In particular, taking $y=x$,

$$
T\left(\alpha_{1}(e, x, x), \alpha_{1}\left(h^{-1}, x, z\right)\right)=\alpha_{1}\left(h^{-1}, x, z\right) \leq \alpha_{1}(h, z, x)
$$

and

$$
S\left(\alpha_{2}(e, x, x), \alpha_{2}\left(h^{-1}, x, z\right)\right)=\alpha_{2}\left(h^{-1}, x, z\right) \geq \alpha_{2}(h, z, x)
$$

and the result follows by symmetry.

$\alpha(e, x, y)$ provides the degree to which $y$ is the transformed of $x$ by the identity element $e$ of $G$. It will then measure the degree in which we can consider $x$ and $y$ as equivalent or indistinguishable objects and will reflect the granularity on $I$. In fact, this relation is a $(T, S)$-indistinguishability operator as it will be proved in Proposition 5.

Definition 15. Let $\alpha$ be an intuitionistic fuzzy action of $G$ on $I . E_{l}=\left(E_{l_{1}}, E_{l_{2}}\right)$ is the intuitionistic fuzzy relation on I defined for all $x, y \in I$ by $E_{l}(x, y)=\alpha(e, x, y)$, i.e., $E_{l_{1}}(x, y)=\alpha_{1}(e, x, y)$ and $E_{l_{2}}(x, y)=\alpha_{2}(e, x, y)$.

Proposition 5. $E_{l}$ is a $(T, S)$-indistinguishability operator.

Proof.

Reflexivity:

$$
E_{l}(x, x)=\alpha(e, x, x)=1_{L^{*}}
$$

Symmetry:

$$
E_{l_{1}}(x, y)=\alpha_{1}(e, x, y)=\alpha_{1}(e, y, x)=E_{l_{1}}(y, x) .
$$

and

$$
E_{l_{2}}(x, y)=\alpha_{2}(e, x, y)=\alpha_{2}(e, y, x)=E_{l_{2}}(y, x) .
$$

Then

$$
E_{l}(x, y)=\alpha(e, x, y)=\alpha(e, y, x)=E_{l}(y, x) .
$$

$\underline{(T, S) \text {-transitivity: }}$

$$
\begin{aligned}
T\left(E_{l_{1}}(x, y), E_{l_{1}}(y, z)\right) & =T\left(\alpha_{1}(e, x, y), \alpha_{1}(e, y, z)\right) \\
& \leq \alpha_{1}(e, x, z)=E_{l_{1}}(x, z)
\end{aligned}
$$

and

$$
\begin{aligned}
S\left(E_{l_{2}}(x, y), E_{l_{2}}(y, z)\right) & =S\left(\alpha_{2}(e, x, y), \alpha_{2}(e, y, z)\right) \\
& \geq \alpha_{2}(e, x, z)=E_{l_{2}}(x, z) .
\end{aligned}
$$

The inequality follows from 3. and 4. in Definition 13

For a crisp action, fixing $g$, the map $f_{g}: I \rightarrow I$ defined by $f_{g}(x)=g x$ is a bijection. The generalization of this result is the next. 
Definition 16. Let $\alpha: G \times I \times I \rightarrow L^{*}$ be an intuitionistic fuzzy action. Fixing $g \in G$, the intuitionistic fuzzy relation $R_{g}: I \times I \rightarrow L^{*}$ is defined for all $x, y \in I$ by

$$
R_{g}(x, y)=\alpha(g, x, y)
$$

Proposition 6. The intuitionistic fuzzy relation $R_{g}=\left(R_{g_{1}}, R_{g_{2}}\right)$ is an injective fuzzy mapping with respect to the $(T, S)$-indistinguishability operator $E_{l}$.

Proof.

$$
\begin{aligned}
T\left(R_{g_{1}}(x, y), E_{l_{1}}\left(x, x^{\prime}\right), E_{l_{1}}\left(y, y^{\prime}\right)\right) & =T\left(\alpha_{1}(g, x, y), \alpha_{1}\left(e, x, x^{\prime}\right), \alpha_{1}\left(e, y, y^{\prime}\right)\right) \\
& =T\left(\alpha_{1}(g, x, y), \alpha_{1}\left(e, x^{\prime}, x\right), \alpha_{1}\left(e, y, y^{\prime}\right)\right) \\
& \leq \alpha_{1}\left(g, x^{\prime}, y^{\prime}\right)=R_{g_{1}}\left(x^{\prime}, y^{\prime}\right)
\end{aligned}
$$

and

$$
\begin{aligned}
S\left(R_{g_{2}}(x, y), E_{l_{2}}\left(x, x^{\prime}\right), E_{l_{2}}\left(y, y^{\prime}\right)\right) & =S\left(\alpha_{2}(g, x, y), \alpha_{2}\left(e, x, x^{\prime}\right), \alpha_{2}\left(e, y, y^{\prime}\right)\right) \\
& =S\left(\alpha_{2}(g, x, y), \alpha_{2}\left(e, x^{\prime}, x\right), \alpha_{2}\left(e, y, y^{\prime}\right)\right) \\
& \geq \alpha_{2}\left(g, x^{\prime}, y^{\prime}\right)=R_{g_{2}}\left(x^{\prime}, y^{\prime}\right) . \\
T\left(R_{g_{1}}(x, y), R_{g_{1}}\left(x, y^{\prime}\right)\right) & =T\left(\alpha_{1}(g, x, y), \alpha_{1}\left(g, x, y^{\prime}\right)\right) \\
& =T\left(\alpha_{1}\left(g^{-1}, y, x\right), \alpha_{1}\left(g, x, y^{\prime}\right)\right) \\
& \leq \alpha_{1}\left(e, y, y^{\prime}\right)=E_{l_{1}}\left(y, y^{\prime}\right)
\end{aligned}
$$

and

$$
\begin{aligned}
S\left(R_{g_{2}}(x, y), R_{g_{2}}\left(x, y^{\prime}\right)\right) & =S\left(\alpha_{2}(g, x, y), \alpha_{2}\left(g, x, y^{\prime}\right)\right) \\
& =S\left(\alpha_{2}\left(g^{-1}, y, x\right), \alpha_{2}\left(g, x, y^{\prime}\right)\right) \\
& \geq \alpha_{2}\left(e, y, y^{\prime}\right)=E_{l_{2}}\left(y, y^{\prime}\right) .
\end{aligned}
$$

Injectivity:

$$
\begin{aligned}
T\left(R_{g_{1}}(x, y), R_{g_{1}}\left(x^{\prime}, y^{\prime}\right) E_{\alpha_{1}}\left(y, y^{\prime}\right)\right) & =T\left(\alpha_{1}(g, x, y), \alpha_{1}\left(g, x^{\prime}, y^{\prime}\right), E_{\alpha_{1}}\left(y, y^{\prime}\right)\right) \\
& =T\left(\alpha_{1}(g, x, y), \alpha_{1}\left(g^{-1}, y^{\prime}, x\right), \alpha_{1}\left(e, y, y^{\prime}\right)\right) \\
& \leq T\left(\alpha_{1}\left(g, x, y^{\prime}\right), \alpha_{1}\left(g^{-1}, y^{\prime}, x^{\prime}\right)\right) \\
& \left.\leq \alpha_{1}\left(e, x, x^{\prime}\right)=E_{l_{1}}\left(x, x^{\prime}\right)\right)
\end{aligned}
$$

and

$$
\begin{aligned}
S\left(R_{g_{2}}(x, y), R_{g_{2}}\left(x^{\prime}, y^{\prime}\right) E_{\alpha_{2}}\left(y, y^{\prime}\right)\right) & =S\left(\alpha_{2}(g, x, y), \alpha_{2}\left(g, x^{\prime}, y^{\prime}\right), E_{\alpha_{2}}\left(y, y^{\prime}\right)\right) \\
& =S\left(\alpha_{2}(g, x, y), \alpha_{2}\left(g^{-1}, y^{\prime}, x\right), \alpha_{2}\left(e, y, y^{\prime}\right)\right) \\
& \geq S\left(\alpha_{2}\left(g, x, y^{\prime}\right), \alpha_{2}\left(g^{-1}, y^{\prime}, x^{\prime}\right)\right) \\
& \left.\geq \alpha_{2}\left(e, x, x^{\prime}\right)=E_{l_{2}}\left(x, x^{\prime}\right)\right) .
\end{aligned}
$$


Proposition 7. $R_{g}$ and $R_{g^{-1}}$ are inverse mappings.

Proof.

$$
\begin{aligned}
T\left(R_{g_{1}}(x, y), E_{l_{1}}\left(y, y^{\prime}\right) R_{g_{1}^{-1}}\left(y^{\prime}, x^{\prime}\right)\right) & =T\left(\alpha_{1}(g, x, y), \alpha_{1}\left(e, y, y^{\prime}\right), \alpha_{1}\left(g^{-1}, y^{\prime}, x^{\prime}\right)\right) \\
& \leq T\left(\alpha_{1}(g, x, y), \alpha_{1}\left(g^{-1}, y, x^{\prime}\right)\right) \\
& \left.\leq \alpha_{1}\left(e, x, x^{\prime}\right)=E_{l_{1}}\left(x, x^{\prime}\right)\right)
\end{aligned}
$$

and

$$
\begin{aligned}
S\left(R_{g_{2}}(x, y), E_{l_{2}}\left(y, y^{\prime}\right) R_{g_{2}^{-1}}\left(y^{\prime}, x^{\prime}\right)\right) & =S\left(\alpha_{2}(g, x, y), \alpha_{2}\left(e, y, y^{\prime}\right), \alpha_{2}\left(g^{-1}, y^{\prime}, x^{\prime}\right)\right) \\
& \geq S\left(\alpha_{2}(g, x, y), \alpha_{2}\left(g^{-1}, y, x^{\prime}\right)\right) \\
& \left.\geq \alpha_{2}\left(e, x, x^{\prime}\right)=E_{l_{2}}\left(x, x^{\prime}\right)\right) . \\
T\left(R_{g_{1}^{-1}}(y, x), E_{l_{1}}\left(x, x^{\prime}\right), R_{g_{1}}\left(x^{\prime}, y^{\prime}\right)\right) & =T\left(\alpha_{1}\left(g^{-1}, y, x\right), \alpha_{1}\left(e, x, x^{\prime}\right), \alpha_{1}\left(g, x^{\prime}, y^{\prime}\right)\right) \\
& \leq T\left(\alpha_{1}\left(g^{-1}, y, x^{\prime}\right), \alpha_{1}\left(g, x^{\prime}, y^{\prime}\right)\right) \\
& \left.\leq \alpha_{1}\left(e, y, y^{\prime}\right)=E_{l_{1}}\left(y, y^{\prime}\right)\right)
\end{aligned}
$$

and

$$
\begin{aligned}
S\left(R_{g_{2}^{-1}}(y, x), E_{l_{2}}\left(x, x^{\prime}\right), R_{g_{2}}\left(x^{\prime}, y^{\prime}\right)\right) & =S\left(\alpha_{2}\left(g_{1}^{-1}, y, x\right), \alpha_{2}\left(e, x, x^{\prime}\right), \alpha_{2}\left(g, x^{\prime}, y^{\prime}\right)\right) \\
& \geq S\left(\alpha_{2}\left(g^{-1}, y, x^{\prime}\right), \alpha_{2}\left(g, x^{\prime}, y^{\prime}\right)\right) \\
& \left.\geq \alpha_{2}\left(e, y, y^{\prime}\right)=E_{l_{2}}\left(y, y^{\prime}\right)\right) .
\end{aligned}
$$

In a (crisp) action, we intend to consider as equivalent the elements that are equal but for the action of an element $g \in G$, so that two elements $x$ and $y$ of $I$ are considered equivalent if and only if there exists $g \in G$ such that $y=g x$. The next definition fuzzifies this idea.

Definition 17. Given an intuitionistic fuzzy action $\alpha$, we define the intuitionistic fuzzy relation $E_{\alpha}=\left(E_{\alpha_{1}}, E_{\alpha_{2}}\right)$ on $I$ by $E_{\alpha_{1}}(x, y)=\sup _{g \in G} \alpha(g, x, y)$ and $E_{\alpha_{2}}(x, y)=\inf _{g \in G} \alpha(g, x, y)$ for all $x, y \in I$.

Proposition 8. If $T$ is a left continuous $t$-norm, then $E_{\alpha}$ is a $(T, S)$-indistinguishability operator on $I$.

Proof.

Reflexivity:

$$
E_{\alpha_{1}}(x, x)=\sup _{g \in G} \alpha_{1}(g, x, x) \geq \alpha_{1}(e, x, x)=1
$$

and

$$
E_{\alpha_{2}}(x, x)=\inf _{g \in G} \alpha_{2}(g, x, x) \leq \alpha_{2}(e, x, x)=0 .
$$


Symmetry:

$$
E_{\alpha_{1}}(y, x)=\sup _{g \in G} \alpha_{1}(g, y, x)=\sup _{g \in G} \alpha_{1}\left(g^{-1}, x, y\right)=\sup _{g \in G} \alpha_{1}(g, x, y)=E_{\alpha_{1}}(x, y)
$$

and

$$
E_{\alpha_{2}}(y, x)=\sup _{g \in G} \alpha_{2}(g, y, x)=\inf _{g \in G} \alpha_{2}\left(g^{-1}, x, y\right)=\inf _{g \in G} \alpha_{2}(g, x, y)=E_{\alpha_{2}}(x, y) .
$$

$\underline{(T, S) \text {-transitivity: }}$

$$
\begin{aligned}
T\left(E_{\alpha_{1}}(x, y), E_{\alpha_{1}}(y, z)\right) & =T\left(\sup _{g \in G} \alpha_{1}(g, x, y), \sup _{h \in G} \alpha_{1}(h, y, z)\right) \\
& =\sup _{g, h \in G} T\left(\alpha_{1}(g, x, y), \alpha_{1}(h, y, z)\right) \\
& \leq \sup _{g, h \in G} \alpha_{1}(h g, x, z) \\
& =E_{\alpha_{1}}(x, z)
\end{aligned}
$$

and

$$
\begin{aligned}
S\left(E_{\alpha_{2}}(x, y), E_{\alpha_{2}}(y, z)\right) & =T\left(\inf _{g \in G} \alpha_{2}(g, x, y), \inf _{h \in G} \alpha_{2}(h, y, z)\right) \\
& =\inf _{g, h \in G} S\left(\alpha_{2}(g, x, y), \alpha_{2}(h, y, z)\right) \\
& \geq \inf _{g, h \in G} \alpha_{2}(h g, x, z) \\
& =E_{\alpha_{2}}(x, z)
\end{aligned}
$$

The inequalities follows from 3. and 4. in Definition 13.

Definition 18. Fixing $x \in I$, the columns $\mu_{x}$, and $\mu_{x}$, of $E_{\alpha}$ (i.e., the intuitionistic fuzzy set $\left.\nu_{x}(y)=E_{\alpha_{1}}(x, y), \nu_{x}(y)=E_{\alpha_{2}}(x, y)\right)$ is the intuitionistic fuzzy orbit of $x$.

The intuitionistic fuzzy orbit of $x \in I$ is therefore the intuitionistic fuzzy equivalence class of $x$ with respect to $E_{\alpha}$.

Proposition 9. Let $\alpha=\left(\alpha_{1}, \alpha_{2}\right)$ such that $\alpha_{1}: G \times I \times I \rightarrow[0,1]$ and $\alpha_{2}: G \times I \times I \rightarrow$ $[0,1]$ be an intuitionistic fuzzy action. For each $g \in G$, the intuitionistic fuzzy relation $R_{g}$ is an injective intuitionistic fuzzy mapping with respect to the $T, S$-indistinguishability operator $E_{\alpha}=\left(E_{\alpha_{1}}, E_{\alpha_{2}}\right)$.

Proof.

$$
\begin{aligned}
T\left(R_{g_{1}}(x, y), E_{\alpha_{1}}\left(x, x^{\prime}\right), E_{\alpha_{1}}\left(y, y^{\prime}\right)\right) & \leq T\left(\sup _{l \in G} \alpha_{1}(l, x, y), \sup _{h \in G} \alpha_{1}\left(h, x, x^{\prime}\right), \sup _{k \in G} \alpha_{1}\left(k, y, y^{\prime}\right)\right) \\
& =T\left(E_{\alpha_{1}}(x, y), E_{\alpha_{1}}\left(x, x^{\prime}\right), E_{\alpha_{1}}\left(y, y^{\prime}\right)\right) \\
& \leq E_{\alpha_{1}}\left(x^{\prime}, y^{\prime}\right)
\end{aligned}
$$

and

$$
\begin{aligned}
S\left(R_{g_{2}}(x, y), E_{\alpha_{2}}\left(x, x^{\prime}\right), E_{\alpha_{2}}\left(y, y^{\prime}\right)\right) & \geq S\left(\inf _{l \in G} \alpha_{2}(l, x, y), \inf _{h \in G} \alpha_{2}\left(h, x, x^{\prime}\right), \inf _{k \in G} \alpha_{2}\left(k, y, y^{\prime}\right)\right) \\
& =S\left(E_{\alpha_{2}}(x, y), E_{\alpha_{2}}\left(x, x^{\prime}\right), E_{\alpha_{2}}\left(y, y^{\prime}\right)\right) \\
& \geq E_{\alpha_{2}}\left(x^{\prime}, y^{\prime}\right) .
\end{aligned}
$$




$$
\begin{aligned}
T\left(R_{g_{1}}(x, y), R_{g_{1}}\left(x, y^{\prime}\right)\right) & =T\left(\alpha_{1}(g, x, y), \alpha_{1}\left(g, x, y^{\prime}\right)\right) \\
& \leq T\left(E_{\alpha_{1}}\left(x, x^{\prime}\right), E_{\alpha_{1}}\left(x, y^{\prime}\right)\right) \\
& \leq E_{\alpha_{1}}\left(y, y^{\prime}\right)
\end{aligned}
$$

and

$$
\begin{aligned}
S\left(R_{g_{2}}(x, y), R_{g_{2}}\left(x, y^{\prime}\right)\right) & =S\left(\alpha_{2}(g, x, y), \alpha_{2}\left(g, x, y^{\prime}\right)\right) \\
& \geq S\left(E_{\alpha_{2}}\left(x, x^{\prime}\right), E_{\alpha_{2}}\left(x, y^{\prime}\right)\right) \\
& \geq E_{\alpha_{2}}\left(y, y^{\prime}\right) .
\end{aligned}
$$

Injectivity:

$$
\begin{aligned}
T\left(R_{g_{1}}(x, y), R_{g_{1}}(z, t), E_{\alpha_{1}}(y, t)\right) & =T\left(\alpha_{1}(g, x, y), \alpha_{1}(g, z, t), E_{\alpha_{1}}(y, t)\right) \\
& \leq T\left(E_{\alpha_{1}}(x, y), E_{\alpha_{1}}(z, t), E_{\alpha_{1}}(y, t)\right) \\
& \leq E_{\alpha_{1}}(x, z)
\end{aligned}
$$

and

$$
\begin{aligned}
S\left(R_{g_{2}}(x, y), R_{g_{2}}(z, t), E_{\alpha_{2}}(y, t)\right) & =S\left(\alpha_{2}(g, x, y), \alpha_{2}(g, z, t), E_{\alpha_{2}}(y, t)\right) \\
& \geq S\left(E_{\alpha_{2}}(x, y), E_{\alpha_{2}}(z, t), E_{\alpha_{2}}(y, t)\right) \\
& \geq E_{\alpha_{2}}(x, z) .
\end{aligned}
$$

Considering an action $\alpha$ on a set $I$, useful (intuitionistic fuzzy or crisp) relations on $I$ should be compatible with it in the sense that they should be invariant under the effect of the action $\alpha$.

Definition 19. Let $\alpha$ be a crisp action on I. An intuitionistic fuzzy relation $R$ on $I$ is invariant under $\alpha$ if and only if

$$
R(x, y)=R(\alpha(g, x), \alpha(g, y))
$$

for all $g \in G, x, y \in I$.

For intuitionistic fuzzy actions, the previous definition can be generalized as follows.

Definition 20. Let $\alpha$ be an intuitionistic fuzzy action of $G$ on $I$ and $R$ be an intuitionistic fuzzy relation on $I . R$ is invariant under $\alpha$ if and only if

$$
T\left(R_{1}(x, y), \alpha_{1}\left(g, x, x^{\prime}\right), \alpha_{1}\left(g, y, y^{\prime}\right)\right) \leq R_{1}\left(x^{\prime}, y^{\prime}\right)
$$

and

$$
S\left(R_{2}(x, y), \alpha_{2}\left(g, x, x^{\prime}\right), \alpha_{2}\left(g, y, y^{\prime}\right)\right) \geq R_{2}\left(x^{\prime}, y^{\prime}\right)
$$

for all $g \in G, x, y, x^{\prime}, y^{\prime} \in I$. 
Proposition 10. $E_{l}$ is invariant under $\alpha$.

Proof.

$$
\begin{aligned}
T\left(E_{l_{1}}(x, y), \alpha_{1}\left(g, x, x^{\prime}\right), \alpha_{1}\left(g, y, y^{\prime}\right)\right) & =T\left(\alpha_{1}(e, x, y), \alpha_{1}\left(g, x, x^{\prime}\right), \alpha_{1}\left(g, y, y^{\prime}\right)\right) \\
& \leq T\left(\alpha_{1}\left(g, x, y^{\prime}\right) \alpha_{1}\left(g, x, x^{\prime}\right)\right) \\
& =T\left(\alpha_{1}\left(g^{-1}, y^{\prime}, x\right), \alpha_{1}\left(g, x, x^{\prime}\right)\right) \\
& \leq \alpha_{1}\left(e, y^{\prime}, x^{\prime}\right)=E_{l_{1}}\left(x^{\prime}, y^{\prime}\right)
\end{aligned}
$$

and

$$
\begin{aligned}
S\left(E_{l_{2}}(x, y), \alpha_{2}\left(g, x, x^{\prime}\right), \alpha_{2}\left(g, y, y^{\prime}\right)\right) & =S\left(\alpha_{2}(e, x, y), \alpha_{2}\left(g, x, x^{\prime}\right), \alpha_{2}\left(g, y, y^{\prime}\right)\right) \\
& \geq S\left(\alpha_{2}\left(g, x, y^{\prime}\right) \alpha_{2}\left(g, x, x^{\prime}\right)\right) \\
& =S\left(\alpha_{2}\left(g^{-1}, y^{\prime}, x\right), \alpha_{2}\left(g, x, x^{\prime}\right)\right) \\
& \geq \alpha_{2}\left(e, y^{\prime}, x^{\prime}\right)=E_{l_{2}}\left(x^{\prime}, y^{\prime}\right) .
\end{aligned}
$$

Proposition 11. $E_{l}$ is the smallest $(T, S)$-indistinguishability operator on $X$ invariant under $\alpha$.

Proof. If $E$ is a $(T, S)$-indistinguishability operator on $I$ invariant under $\alpha$, then for all $x, x^{\prime}, y$, $y^{\prime} \in I$ and $g \in G$,

$$
T\left(E_{1}(x, y), \alpha_{1}\left(g, x, x^{\prime}\right), \alpha_{1}\left(g, y, y^{\prime}\right)\right) \leq E_{1}\left(x^{\prime}, y^{\prime}\right)
$$

and

$$
S\left(E_{2}(x, y), \alpha_{2}\left(g, x, x^{\prime}\right), \alpha_{2}\left(g, y, y^{\prime}\right)\right) \geq E_{2}\left(x^{\prime}, y^{\prime}\right) \text {. }
$$

In particular,

$$
\begin{gathered}
T\left(E_{1}(x, y), \alpha_{1}\left(e, x, x^{\prime}\right), \alpha_{1}\left(e, y, y^{\prime}\right)\right) \leq E_{1}\left(x^{\prime}, y^{\prime}\right) \\
T\left(E_{1}(x, y), E_{l_{1}}\left(x, x^{\prime}\right), E_{l_{1}}\left(y, y^{\prime}\right), \leq E_{1}\left(x^{\prime}, y^{\prime}\right)\right.
\end{gathered}
$$

and

$$
\begin{gathered}
S\left(E_{2}(x, y), \alpha_{2}\left(e, x, x^{\prime}\right), \alpha_{2}\left(e, y, y^{\prime}\right)\right) \geq E_{2}\left(x^{\prime}, y^{\prime}\right) \\
S\left(E_{2}(x, y), E_{l_{2}}\left(x, x^{\prime}\right), E_{l_{2}}\left(y, y^{\prime}\right), \geq E_{2}\left(x^{\prime}, y^{\prime}\right) .\right.
\end{gathered}
$$

Putting $x=y=x^{\prime}$,

$$
\begin{aligned}
& T\left(E_{1}(x, x), E_{l_{1}}(x, x), E_{l_{1}}\left(x, y^{\prime}\right), \leq E_{1}\left(x, y^{\prime}\right)\right. \\
& S\left(E_{2}(x, x), E_{l_{2}}(x, x), E_{l_{2}}\left(x, y^{\prime}\right), \geq E_{2}\left(x, y^{\prime}\right)\right.
\end{aligned}
$$

and hence $E_{l_{1}}\left(x, y^{\prime}\right) \leq E_{2}\left(x, y^{\prime}\right)$ and $E_{l_{2}}\left(x, y^{\prime}\right) \geq E_{2}\left(x, y^{\prime}\right)$.

Nota Bene. In fact, we have only used reflexivity of $E$ in the proof, so that states that $E_{l}$ is smaller than or equal to any reflexive intuitionistic fuzzy relation of $I$ invariant under $\alpha$. 
Proposition 12. $E_{\alpha}$ is invariant under $\alpha$.

Proof. $\alpha_{1}\left(g, x, x^{\prime}\right) \leq E_{\alpha_{1}}\left(x, x^{\prime}\right)$ and $\alpha_{1}\left(g, y, y^{\prime}\right) \leq E_{\alpha_{1}}\left(y, y^{\prime}\right)$ and hence

$$
\begin{aligned}
T\left(E_{\alpha_{1}}(x, y), \alpha_{1}\left(g, x, x^{\prime}\right), \alpha_{1}\left(g, y, y^{\prime}\right)\right) & \leq T\left(E_{\alpha_{1}}(x, y), E_{\alpha_{1}}\left(x, x^{\prime}\right), E_{\alpha_{1}}\left(y, y^{\prime}\right)\right) \\
& \leq E_{\alpha_{1}}\left(x^{\prime}, y^{\prime}\right) .
\end{aligned}
$$

and $\quad \alpha_{2}\left(g, x, x^{\prime}\right) \leq E_{\alpha_{2}}\left(x, x^{\prime}\right)$ and $\alpha_{2}\left(g, y, y^{\prime}\right) \leq E_{\alpha_{1}}\left(y, y^{\prime}\right)$ and hence

$$
\begin{aligned}
S\left(E_{\alpha_{2}}(x, y), \alpha_{2}\left(g, x, x^{\prime}\right), \alpha_{2}\left(g, y, y^{\prime}\right)\right) & \geq S\left(E_{\alpha_{2}}(x, y), E_{\alpha_{2}}\left(x, x^{\prime}\right), E_{\alpha_{2}}\left(y, y^{\prime}\right)\right) \\
& \geq E_{\alpha_{2}}\left(x^{\prime}, y^{\prime}\right) .
\end{aligned}
$$

By the previous result it is easy to demonstrate the following corollary.

Corollary 1. We have $\mathrm{E}_{\mathrm{I}} \leq \mathrm{E}_{\alpha}$.

In Proposition 4 we have proved that a perfect intuitionistic fuzzy action generates a crisp action in a natural way. Reciprocally, from a crisp intuitionistic fuzzy action and a $(T, S)$ indistinguishability operator invariant under this action an intuitionistic fuzzy action can be obtained again in a natural way.

Proposition 13. Let $E$ be a $(T, S)$-indistinguishability operator on a set $X$ invariant under the ( crisp) action $x \rightarrow$ gx of a group $G$ on $I$. The mapping $\alpha: G \times I \times I \rightarrow L^{*}$ defined for all $g \in G$ and for all $x, y \in I$ by $\alpha(g, x, y)=E(g x, y)$ is a perfect intuitionistic fuzzy action of $G$ on $I$. Moreover, $E(x, y)=\alpha(e, x, y)=E_{l}(x, y)$.

Proof. We have

$$
\begin{aligned}
T\left(\alpha_{1}(h g, x, y), \alpha_{1}(g, x, z)\right) & =T\left(E_{1}(h g x, y), E_{1}(g x, z)\right) \\
& =T\left(E_{1}(h g x, y), E_{1}(h g x, h z)\right) \\
& \leq E_{1}(h z, y)=\alpha_{1}(h, z, y) .
\end{aligned}
$$

and

$$
\begin{aligned}
& T\left(\alpha_{1}(g, x, z), \alpha_{1}(h, z, y)\right)=T\left(E_{1}(g x, z), E_{1}(h z, y)\right) \\
&=T\left(E_{1}(h g x, h z), E_{1}(h z, y)\right. \\
& \leq E_{1}(h g x, y)=\alpha_{1}(h g, x, y) . \\
& \alpha_{1}(e, x, x)=1=E_{1}(e x, x)=E_{1}(x, x)=1 .
\end{aligned}
$$

$\alpha$ is perfect.

$\alpha_{1}(g, x, g x)=E_{1}(g x, g x)=1$.

$E_{1}(x, y)=\alpha_{1}(e, x, y)=E_{l_{1}}(x, y)$. 
Proposition 14. Fixing $x \in I$, the intuitionistic fuzzy subset $(\mu, \nu)$ of $G$ defined by

$$
\begin{gathered}
\mu(g)=\alpha_{1}(g, x, x), \\
\nu(g)=\alpha_{2}(g, x, x)
\end{gathered}
$$

is an intuitionistic fuzzy subgroup of $G$ with $\mu(e)=1$ and $\nu(e)=0$, where $e$ is the identity element of $G$.

Proof.

$$
\begin{aligned}
& T(\mu(g), \mu(h))=T\left(\alpha_{1}(g, x, x), \alpha_{1}(h, x, x)\right) \leq \alpha_{1}(g h, x, x)=\mu(g h) \\
& \mu(g)=\alpha_{1}(g, x, x)=\alpha_{1}\left(g^{-1}, x, x\right)=\mu\left(g^{-1}\right) \\
& \mu(e)=\alpha_{1}(e, x, x)=1
\end{aligned}
$$

and

$$
\begin{aligned}
& S(\nu(g), \nu(h))=S\left(\alpha_{2}(g, x, x), \alpha_{2}(h, x, x)\right) \geq \alpha_{2}(g h, x, x)=\nu(g h) \\
& \nu(g)=\alpha_{2}(g, x, x)=\alpha_{2}\left(g^{-1}, x, x\right)=\nu\left(g^{-1}\right) \\
& \nu(e)=\alpha_{2}(e, x, x)=0 .
\end{aligned}
$$

Definition 21. $(\mu, \nu)$ is the isotropy intuitionistic fuzzy subgroup of $x \in I$.

It is easy to prove the following proposition.

Proposition 15. If $(\mu, \nu)$ is the isotropy intuitionistic fuzzy subgroup of $x \in I$, then the intuitionistic fuzzy relation $E_{x}$ on $G$ defined for all $g, h \in G$ by

$$
\begin{aligned}
& E_{x_{1}}(g, h)=\mu\left(g h^{-1}\right), \\
& E_{x_{2}}(g, h)=\nu\left(g h^{-1}\right)
\end{aligned}
$$

is a $(T, S)$-indistinguishability operator on $G$.

Proposition 16. Let $\alpha$ be an intuitionistic fuzzy action of $G$ on I. If the t-norm is left continuous, then the intuitionistic fuzzy subset $(\mu, \nu)$ of $G$ defined by

$$
\begin{aligned}
& \mu(g)=\inf _{x \in I} \alpha_{1}(g, x, x), \\
& \nu(g)=\sup _{x \in I} \alpha_{2}(g, x, x)
\end{aligned}
$$

is an intuitionistic fuzzy subgroup of $G$ with $(\mu, \nu)(e)=1_{L^{*}}$ where $e$ is the identity element of $G$.

Proof.

$$
\begin{aligned}
T(\mu(g), \mu(h)) & =T\left(\inf _{x \in I} \alpha_{1}(g, x, x), \inf _{y \in I} \alpha_{1}(h, y, y)\right) \\
& =\inf _{x, y \in I} T\left(\alpha_{1}(g, x, x), \alpha_{1}(h, y, y)\right) \\
& \leq \inf _{x \in I} T\left(\alpha_{1}(g, x, x), \alpha_{1}\left(h^{-1}, x, x\right)\right) \\
& \leq \inf _{x \in I} \alpha_{1}\left(g h^{-1}, x, x\right)=\mu\left(g h^{-1}\right) . \\
\mu(e) & =\inf _{x \in I} \alpha_{1}(e, x, x)=1
\end{aligned}
$$

and 


$$
\begin{aligned}
S(\nu(g), \nu(h)) & =S\left(\sup _{x \in I} \alpha_{2}(g, x, x), \sup _{y \in I} \alpha_{2}(h, y, y)\right) \\
& =\sup _{x, y \in I} T\left(\alpha_{2}(g, x, x), \alpha_{2}(h, y, y)\right) \\
& \geq \sup _{x \in I} T\left(\alpha_{2}(g, x, x), \alpha_{2}\left(h^{-1}, x, x\right)\right) \\
& \geq \sup _{x \in I} \alpha_{2}\left(g h^{-1}, x, x\right)=\nu\left(g h^{-1}\right) . \\
\nu(e) & =\sup _{x \in I} \alpha_{2}(e, x, x)=0 .
\end{aligned}
$$

\section{Conclusion}

This work completes some properties of the concept of intuitionistic fuzzy action already proposed in [6].

\section{References}

[1] Atanassov, K. (1983) Intuitionistic fuzzy sets, VII ITKR Session, Sofia, 20-23 June 1983 (Deposed in Centr. Sci.-Techn. Library of the Bulg. Acad. of Sci., 1697/84) (in Bulgarian). Reprinted: Int. J. Bioautomation, 2016, 20(S1), S1-S6.

[2] Atanassov, K. (1986) Intuitionistic fuzzy sets, Fuzzy Sets and Systems, 20, 87-96.

[3] Boixader, D., \& Recasens, J. (2018) Fuzzy actions, Fuzzy Sets and Systems, 339, 17-30.

[4] Fathi, M. \& Saleh, A. R. (2009) Intuitionistic fuzzy group, Asian Journal of Algebra, 2 (1), $1-10$.

[5] Hur, K., Jang, S. Y. \& Ahn, Y. S. (2005) Intuitionistic fuzzy equivalence relations, Honam Math. J., 27 (2), 163-181.

[6] Tarsuslu, S., Tarsuslu, A. \& Citil, M. (2018) Intuitionistic fuzzy action of a group on a set, Notes on Intuitionistic Fuzzy Sets, 24 (2), 18-24.

[7] Dudek, W. A., Zhan, J., \& Davvaz, B. (2008) Intuitionistic $(S, T)$-fuzzy hyperquasigroups, Soft Comput., 12, 1229-1238. 